\title{
Changes in Esodeviation after Inferior Oblique Recession in Patients with Refractive Accommodative Esotropia and Inferior Oblique Overaction
}

\author{
Donghun Lee ${ }^{1}$, Won Jae Kim², Myung-Mi Kim³ \\ ${ }^{I}$ Department of Ophthalmology, Catholic University of Daegu, School of Medicine, Daegu, Korea \\ ${ }^{2}$ Department of Ophthalmology, Yeungnam University College of Medicine, Daegu, Korea \\ ${ }^{3}$ Nune Eye Hospital, Daegu, Korea
}

\begin{abstract}
Purpose: To evaluate the changes in esodeviation after inferior oblique (IO) recession in patients with refractive accommodative esotropia and 10 overaction.

Methods: Graded 10 recession was performed in 68 patients who were diagnosed with refractive accommodative esotropia with 10 overaction. The patients were followed for at least 3 months after surgery and the angle of esodeviation with correction was evaluated at distance and near at each follow-up evaluation. The patients were divided into two groups: patients who underwent unilateral IO recession (UIO-Rec) and patients who underwent bilateral IO recession (BIO-Rec). The change in esodeviation after surgery was compared between the two groups.

Results: A total of 68 patients were enrolled in this study, with 38 patients in the UIO-Rec group and 30 in the BIO-Rec group. In the UIO-Rec group, there was no statistically significant difference in esodeviation before and after surgery. In the BIO-Rec group, esodeviation at distance increased significantly 1 day postoperatively $(p=0.033)$. However, esodeviation returned to the preoperative value one week after surgery $(p=0.665)$. Changes in esodeviation at distance were significantly greater in the BIO-Rec group than in the UIO-Rec group one day after surgery $(p=0.044)$.

Conclusions: Bilateral IO-weakening surgery induced a transient increase in esodeviation in patients with refractive accommodative esotropia. However, we found no evidence that well-controlled esotropia with corrected hyperopia became decompensated after IO-weakening surgery, as induced esodeviation was minor and temporary.
\end{abstract}

Key Words: Esotropia, Strabismus, Surgery

The primary action of the inferior oblique (IO) muscle is extorsion, with elevation as a secondary action, and abduction as a tertiary action. Because the IO is the only muscle

Received: December 2, 2019 Final revision: April 6, 2020

Accepted: April 23, 2020

Corresponding Author: Myung-Mi Kim, MD, PhD. Nune Eye Hospital, 2179 Dalgubeol-daero, Jung-gu, Daegu 41940, Korea. Tel: 82-53-715-

3300, Fax: 82-53-715-3443, E-mail: mmk@med.yu.ac.kr that can elevate the eye when it is fully adducted, strabismus characterized by "elevation in adduction" is commonly referred to as inferior oblique overaction (IOOA) [1]. Surgical procedures to correct IOOA include myectomy, myotomy, anterior transposition, recession, denervation, and extirpation [2]. Each of these techniques have distinct pros and cons and their reported efficacies vary [3-5], and a result they are used selectively according to the clini- 
cian's experience and preference. Among IO-weakening procedures, graded IO recession is commonly performed because of the ease of reoperation in the event of recurrence and the possibility of graded surgery according to the severity of the IOOA $[5,6]$.

Urist [7] initially reported that combining IO-weakening surgery with concurrent horizontal muscle surgery had very little effect on horizontal deviation, but many subsequent studies reported weakened abduction of the IO muscle. Some studies have reported no significant changes in horizontal deviation after IO-weakening surgery [7-9], while others reported variable effects on horizontal deviation [10-12].

The study populations in many previous studies included patients with exotropia, orthotropia, and/or esotropia $[8,10,12]$. Hence, in previous studies, topics about the horizontal aligning effect of the IO muscle have mostly focused on planning concurrent horizontal muscle surgeries $[8,10]$. To the best of our knowledge, no previous studies have reported the effects of IO muscle weakening on horizontal deviation in patient with only refractive accommodative esotropia. Therefore, in this study, we aimed to evaluate the changes in esodeviation after graded IO recession in patients with refractive accommodative esotropia.

\section{Materials and Methods}

We performed a retrospective analysis of data from patients older than 2 years of age who were diagnosed with refractive accommodative esotropia combined with IOOA in the ophthalmologic clinic at Yeungnam University Medical Center from January 1995 to April 2017 and subsequently underwent graded IO recession. This retrospective study was approved by the institutional review board (2017-11-003) and all procedures adhered to the tenets of the Declaration of Helsinki. Because this is a retrospective study, the study was exempt from patient consent. Patients who underwent concurrent rectus muscle surgery were excluded, as were patients with a history of previous ocular surgery including extraocular muscle surgery. In addition, patients with a high accommodative convergence/accommodation ratio who needed bifocal glasses and patients with partially accommodative esotropia who needed concurrent rectus muscle surgery were also excluded from this study.
Refractive accommodative esotropia was diagnosed if esotropia was eliminated or decreased to within 10 diopters (D) with full cycloplegic hyperopic correction on the first follow-up evaluation at least 1 month after the prescription of spectacles.

Primary IOOA is characterized clinically as overelevation in adduction in the absence of other findings such as positive head tilt or cyclotorsion. Additionally, IOOA secondary to superior oblique palsy is classified as secondary IOOA. The severity of IOOA was graded (while the patient was wearing their hyperopic spectacles) according to the degree of overelevation of the eye in adduction using a scale ranging from +1 to +4 , as described by Rosenbaum and Santiago [13]. The indications for surgery were consistent with those provided by Wright [14]. Patients with +2 or more IOOA were candidates for surgery. In addition, patients with minimal IOOA, but a significant V-pattern ( $>15$ prism diopters [PD]) also underwent surgery. Although clinicians do not typically operate for primary IOOA alone, the authors performed IO recession on patients with primary IOOA in specific cases where patients or their parents wanted a cosmetic improvement in lateral gaze.

Every surgery was performed by a single surgeon (MMK) under general anesthesia. Using the fornix approach, all patients underwent IO muscle surgery using an eyelid speculum with flanges on the blades. After the IO muscle was hooked with a single hook, careful exploration was performed posteriorly along the globe in order to locate any posterior fibers for complete isolation of the muscle. After the IO muscle was disinserted with the Stevens tenotomy scissors and secured with a double-arm suture, exaggerated traction tests were performed to confirm the

Table 1. Surgical amount of graded IO recession

\begin{tabular}{cc}
\hline Grade of IOOA & Amount of IO recession $(\mathrm{mm})$ \\
\hline+2 & $8^{*}$ \\
+3 & $10^{\dagger}$ \\
+4 & $14^{\star}$ \\
\hline
\end{tabular}

$\mathrm{IO}=$ inferior oblique; IOOA = inferior oblique overaction.

"The IO muscle was reattached to the sclera $3 \mathrm{~mm}$ posterior and $4 \mathrm{~mm}$ lateral to the lateral border of the inferior rectus muscle insertion (Classic Fink's point); ${ }^{\dagger}$ The IO muscle was reattached 3 $\mathrm{mm}$ posterior and $2 \mathrm{~mm}$ lateral to the lateral border of the inferior rectus muscle insertion (Scheie-Parks point); ${ }^{*}$ The IO muscle was reattached near the inferotemporal vortex vein. 


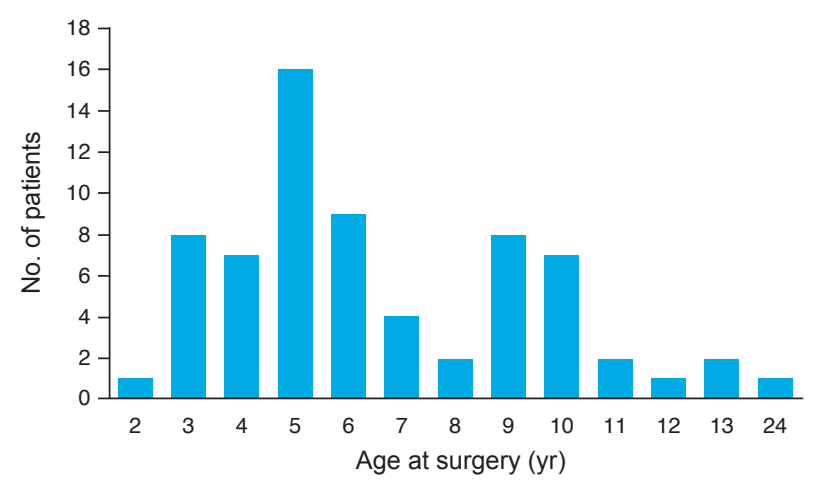

Fig. 1. The mean age at surgery of 68 patients was 6.6 years.
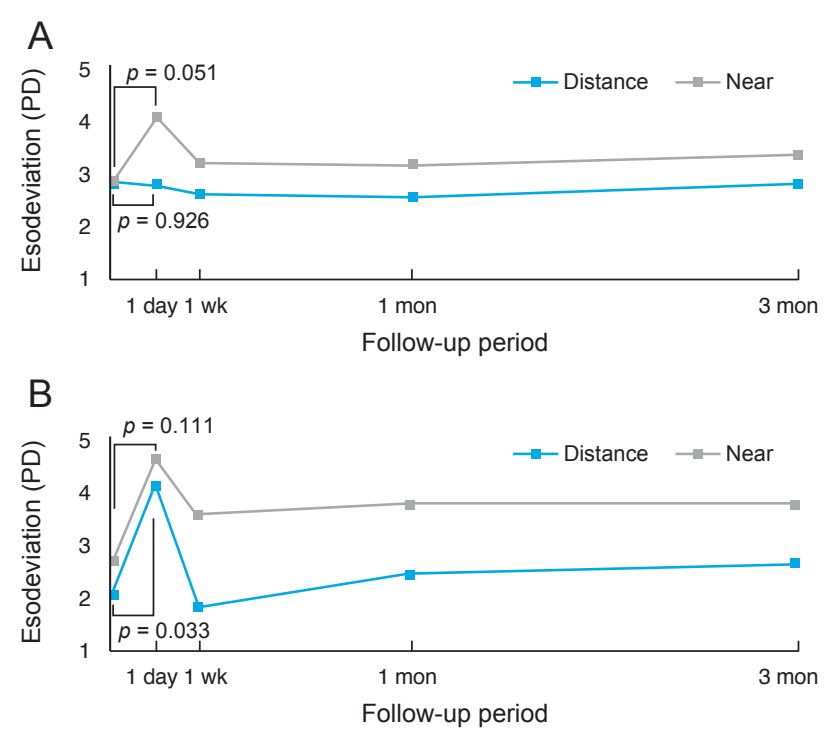

Fig. 2. Changes in esodeviation after surgery (paired $t$-test). (A) The patients who underwent unilateral graded IO recession. (B) The patients who underwent bilateral graded $\mathrm{IO}$ recession. $\mathrm{PD}=$ prism diopters.

entire IO muscle had been cut. The muscle was reattached to the globe at the desired point. The amount of graded IO recession was based on the surgical tables presented in Table 1 . The technique described above was a modification of the method described by Parks and Parker [15] and Fink [16]. The occurrence of IOOA in the opposite eye after unilateral IO surgery and the recurrence of IOOA in the operated eye were considered present if IOOA greater than grade +2 was observed.

In all cases, cycloplegic refraction was determined before surgery. Refractive errors were measured 30 minutes after instilling $1 \%$ cyclopentolate three times at 5-minute intervals, and the results were converted to a spherical equivalent. Stereoacuity was measured using the Lang I stereotest (Lang-Stereotest AG, Küsnacht, Switzerland) and the Stereo Fly Stereotest (Stereo Optical Co., Chicago, IL, USA) in cooperative patients. The angle of esodeviation with correction was evaluated using the alternate prism cover test at distance $(6 \mathrm{~m})$ and near $(33 \mathrm{~cm})$ preoperatively and at 1 day, 1 week, 1 month and 3 months postoperatively. The angles of deviation in upgaze and downgaze were also measured to assess significant V-patterns, but angles used for the statistical analyses were all measured at the primary position. In order to exclude factors that measuring postoperative angles in unintentional slightly downgaze might misidentify the changes in deviation to esotropia, the clinicians tried to measure the angles under the same conditions as far as possible; the patient's head was stabilized on the chinrest and headrest. Manifesting the deviation was completed after the full hyperopic correction on the basis of their cycloplegic refraction.

The enrolled patients were divided into two groups: the patients who underwent unilateral graded IO recession (UIO-Rec group) and the patients who underwent bilateral graded IO recession (BIO-Rec group). Baseline characteristics and changes in esodeviation after surgery were compared between the two groups. Statistical analyses were performed using PASW Statistics ver. 18.0 (SPSS Inc., Chicago, IL, USA). The paired $t$-test was used to analyze the changes in esodeviation after surgery in each group and the independent $t$-test was used to compare preoperative factors between the two groups. A $p$-value of less than 0.05 was considered statistically significant.

\section{Results}

A total of 68 patients (27 male and 41 female patients) met the inclusion criteria for this study. Before IO surgery, the mean esotropia without hyperopic correction was 22.78 PD (range, 10 to 45 PD) at distance and the mean magnitude of esotropia reduction after hyperopic correction was 19.38 PD (range, 4 to 45 PD). Fig. 1 presents the distributions of the patient ages at surgery. The median age at the time of surgery was 6 years (range, 2 to 24 years). The median follow-up period was 8.50 years (range, 0.5 to 24 years). The mean preoperative esodeviation with correction was $2.31 \pm 3.40 \mathrm{PD}$ (range, 0 to $10 \mathrm{PD}$ ) at distance and $2.59 \pm 3.65 \mathrm{PD}$ (range, 0 to $14 \mathrm{PD}$ ) at near. In addition, me- 
Table 2. Baseline characteristics of the patients

\begin{tabular}{|c|c|c|c|}
\hline & UIO-Rec group & BIO-Rec group & $p$-value \\
\hline No. pf patients & 38 & 30 & \\
\hline Male : female & $17: 21$ & $10: 20$ & $0.340^{*}$ \\
\hline Age at first visit (yr) & $3.84 \pm 3.93$ & $2.47 \pm 2.26$ & $0.093^{\dagger}$ \\
\hline Age at surgery (yr) & $7.42 \pm 3.84$ & $5.87 \pm 2.71$ & $0.065^{\dagger}$ \\
\hline Mean follow-up period (yr) & $8.10 \pm 4.97$ & $9.76 \pm 6.56$ & $0.240^{\dagger}$ \\
\hline \multicolumn{4}{|l|}{ Type of IOOA } \\
\hline Primary IOOA (IOOA only) & 11 & 13 & $0.306^{*}$ \\
\hline Secondary IOOA (IOOA + SOP) & 27 & 17 & \\
\hline \multicolumn{4}{|c|}{ Preoperative esodeviation with correction (PD) } \\
\hline Distance & $2.82 \pm 3.89$ & $2.07 \pm 3.91$ & $0.434^{\dagger}$ \\
\hline Near & $2.87 \pm 4.26$ & $2.70 \pm 4.13$ & $0.870^{\dagger}$ \\
\hline \multicolumn{4}{|l|}{ Result of stereotest } \\
\hline Lang I test, pass & $5 / 25$ & $2 / 20$ & $0.311^{*}$ \\
\hline Stereo Fly Stereotest, $\leq 400 \operatorname{arcsec}$ & $8 / 25$ & $4 / 20$ & $0.502^{*}$ \\
\hline \multicolumn{4}{|c|}{ Preoperative median SE refractive errors (D) } \\
\hline Right eye & $+2.01 \pm 1.98$ & $+2.22 \pm 2.06$ & $0.671^{\dagger}$ \\
\hline Left eye & $+2.75 \pm 4.16$ & $+2.37 \pm 2.33$ & $0.653^{\dagger}$ \\
\hline
\end{tabular}

Values are presented as number or mean \pm standard deviation.

UIO-Rec group = unilateral graded inferior oblique recession; BIO-Rec group = bilateral graded inferior oblique recession; IOOA = inferior oblique overaction; $\mathrm{SOP}=$ superior oblique palsy; $\mathrm{PD}=$ prism diopters; $\mathrm{SE}=$ spherical equivalent; $\mathrm{D}=$ diopters.

${ }^{*}$ Chi-square test; ${ }^{\dagger}$ Independent $t$-test.

dian preoperative esodeviations with correction were 0 PD for both distance and near. The median preoperative re-

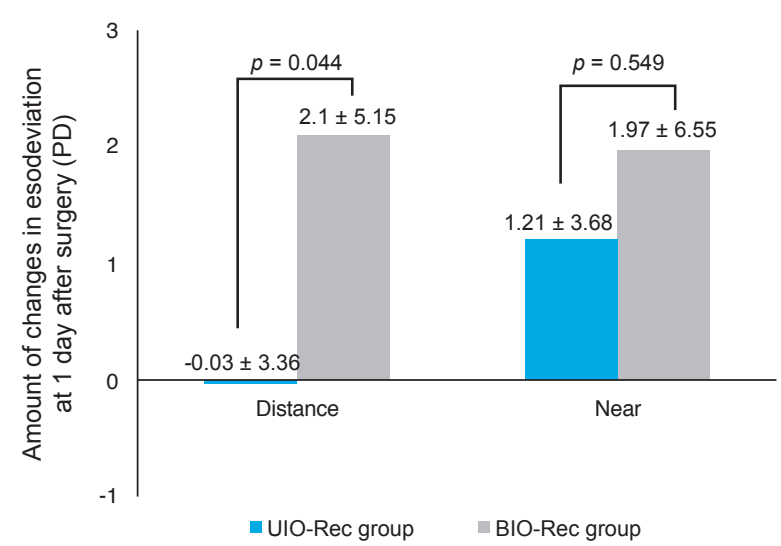

Fig. 3. Comparison of changes in esodeviation between the patients who underwent unilateral graded IO recession (UIO-Rec) and the patients who underwent bilateral graded IO recession (BIO-Rec group) 1 day after surgery (independent $t$-test). PD = prism diopters; + = esodeviation; - = exodeviation. fractive error was $+2.37 \mathrm{D}$ (range, +0.75 to $+7.50 \mathrm{D}$ ) for the more hyperopic eye and $+2.00 \mathrm{D}$ (range, plano to $+6.50 \mathrm{D}$ ) for the less hyperopic eye. Three patients had surgeries due to a V-pattern (>15 PD) with minimal IOOA. Their V-patterns improved after surgery. Twenty-four patients had primary IOOA and 44 had secondary IOOA. Thirty-eight patients were enrolled in the UIO-Rec group (unilateral graded IO recession) and 30 patients were enrolled in the BIO-Rec group (bilateral graded IO recession). The mean grade of IOOA in the UIO-Rec group was +2.9 and the mean grade of IOOA per eye in the BIO-Rec group was +2.7 . There was no significant difference in the mean grade of IOOA per eye between the two groups. A comparison of baseline characteristics between the two groups is provided in Table 2. There were no significant differences in gender, age at first visit, age at surgery, mean follow-up period, spherical equivalent refractive error of each eye, or preoperative esodeviation with correction between the two groups. In addition, there was no difference the percentage 
of patients with primary or secondary IOOA between the two groups. Twenty-five patients in the UIO-Rec group and 20 in the BIO-Rec group were able to cooperate and complete the stereotest. Among them, 5 of 25 patients in UIO-Rec group and two of 20 in BIO-Rec group passed the Lang I test. Furthermore, two of 25 patients in UIORec group and four of 20 patients in BIO-Rec group were within 400 seconds of arc in the Stereo Fly stereotest. There were no statistically significant differences in the results of the two stereotests between the two groups ( $p=$ $0.311, p=0.502$, for the Lang I and Stereo Fly tests, respectively).

One day after surgery, the UIO-Rec group had $2.79 \pm$ 3.24 PD of distance esodeviation and $4.08 \pm 4.00 \mathrm{PD}$ of near esodeviation (Fig. 2A). Compared to the preoperative values, there were no statistically significant differences over the 3-month follow-up period. The occurrence of IOOA in the contralateral eye and recurrence of greater than +2 -grade IOOA in the operated eye were not observed in any patients during the 3-month follow-up. In the BIORec group, $4.17 \pm 4.73$ PD of distance esodeviation and 4.67 $\pm 5.47 \mathrm{PD}$ of near esodeviation were measured one day after surgery (Fig. 2B). Distance esodeviation increased significantly one day after surgery ( $p=0.033$ ) but returned to the preoperative value after one week $(p=0.665)$. Near esodeviation in the two groups did not differ significantly over the 3-month follow-up period. The recurrence of greater than +2 -grade IOOA in the operated eye was not observed in any patient in the 3-month follow-up period.

Esodeviation increased in four patients in the BIO-Rec group one day after surgery and persisted over the 3-month follow-up period. The mean esodeviation in these patients was $6 \pm 4 \mathrm{PD}$ (range, 4 to $12 \mathrm{PD}$ ) after 3 months. Although cycloplegic refraction was repeated and the eyeglasses changed as necessary to correct additional hyperopia (range, +0.125 to $+0.50 \mathrm{D}$ ), esodeviation did not decrease. None of the four patients demonstrated any binocular single vision in either the Lang I or Stereo Fly Stereotest, which may well be a significant factor.

Fig. 3 presents the changes in esodeviation in the two groups 1 day after the IO surgery. Changes in distance esodeviation were significantly larger in the BIO-Rec group than in the UIO-Rec group ( $p=0.044)$. However, changes in near esodeviation were not statistically significant between the two groups ( $p=0.549$ ).

\section{Discussion}

Unlike exotropia and esotropia unrelated to refractive error, refractive accommodative esotropia is caused by several factors, including the relationship between accommodation and convergence, or abnormal activation of the accommodation reflex required to overcome blurring caused by uncorrected hyperopia [17]. Many clinicians have observed various changes in esodeviation of refractive accommodative esotropia such as changes to consecutive exotropia or to decompensated accommodative esotropia [18-21]. Previous studies have suggested the presence of amblyopia, failure to achieve central fusion, and high accommodative convergence/accommodation ratio may be risk factors for changes in deviation [19-21].

Development of IOOA has been reported in 34\% of cases of accommodative esotropia [22]. Given the IO muscle functions as an abductor as well as an elevator, we hypothesized that IO-weakening surgery may affect the change in esodeviation in these patients. Previous studies on changes in esodeviation after IO-weakening surgery [7-12] have produced conflicting results that may have reflected differences in the enrolled patient populations and the surgical procedures used in each study. Taylan Sekeroglu et al. [10] reported $4 \mathrm{PD}$ esoshift in 66 patients regardless of the type of IO-weakening surgery and suggested that this should be considered to plan a simultaneous horizontal muscle surgery in setting the surgical amount. Conversely, Stager and Parks [9] demonstrated that no significant horizontal change occurred in $84 \%$ of the patients with preoperative horizontal deviation of less 10 PD who had bilateral IO myectomy or recession. In addition, Urist [7] reported bilateral IO recession had very little effect on esodeviation when concurrent horizontal muscle surgeries were performed.

However, to our knowledge, no studies have investigated the influence of the IO muscle on horizontal deviation for patients with refractive accommodative esotropia. Refractive accommodative esotropia occurs at a relatively young age, with 18 to 48 months of age [23], and IOOA can develop whether this is primary or secondary, during their longrange observation periods. Some patients may require corrective surgery to address the IO muscle. Therefore, the most important aims of this study were to investigate the function of the IO muscle as an abductor in a specific situation in which excessive accommodation produces esode- 
viation, and to compare the unilateral and bilateral surgery groups to evaluate whether the degree of IO weakening influenced changes in esodeviation. In addition, we aimed to investigate changes in the abduction function of the IO muscle by including only patients who underwent IO-weakening surgery alone and excluding those who underwent concurrent horizontal muscle surgery.

In the present study, one day after surgery, esodeviation increased $2.10 \pm 5.15 \mathrm{PD}$ (range, 0 to $12 \mathrm{PD}$ ) in the BIORec group, similar to changes in esodeviation after IO-weakening surgery reported in previous studies $[10,11]$. Esodeviation in the UIO-Rec group did not change and the change in esodeviation at distance was significantly larger in the BIO-Rec group than in the UIO-Rec group. It is thought that the amount of weakening in abduction of the IO muscle was likely larger in the BIO-Rec group (two IO muscle surgeries in one patient) that the UIO-Rec group (one IO muscle surgery in one patient). As a result, the weakening of abduction in both IO muscles caused a significant esoshift in the primary position only in the BIORec group.

However, the increased esodeviation was not permanent and resolved one week after the surgery in this study. We hypothesize that the increased esodeviation was transient because the IO muscle functions mainly as an elevator and torsioner, in addition to its tertiary function as an abductor [2]. In other words, a procedure to weaken the IO muscle can create a permanent reduction of "elevation in adduction," but reduction of "abduction" may be restored by the action of a main abductor, the lateral rectus muscle.

Four patients in the BIO-Rec group had an increase in esodeviation after surgery, which did not resolve in the postoperative period and persisted through the follow-up period. Stereopsis is an element of binocular single vision and high-grade stereoacuity, and is important in maintaining a normal ocular alignment [24]. Therefore, these patients may have had poor fusional control such that they could not overcome the increase in esodeviation. In addition, all of four patients had secondary IOOA. The superior oblique muscle is also an abductor, and if not already fully functioning, it is possible that completion of a weakening procedure on the IO muscle may have a more significant impact on abduction.

The purpose of this study was to investigate whether IO-weakening surgery may influence esodeviation in a specific situation in which excessive accommodation caus- es the disease (i.e., refractive accommodative esotropia); patients with both primary and secondary IOOA were included in the statistical analyses if they had refractive accommodative esotropia. However, there were no significant differences in the numbers of enrolled patients with primary or secondary of IOOA between the UIO-Rec and BIO-Rec groups (Table 2).

This study had several limitations. It was a retrospective study with a relatively short follow-up period, and the deviation measurements are less accurate in very young patients (our study included patients as young as 2 years of age). In addition, stereotesting could not be completed in some young patients because of poor cooperation, and the number of patients whose increased esodeviation did not resolve over the 3-month follow-up period was too small to demonstrate statistical significance. Further studies would be required to investigate the changes in horizontal deviation after other kinds of IO-weakening surgery, such as IO myectomy and myotomy. Moreover, because the purpose of most of the IO surgeries this study was to achieve stable alignment in lateral gaze and to treat IOOA, information regarding the degree of V-pattern in some patients was missing from the medical records, and the authors could not address the improvement of V-pattern of patients who had IOOA.

In conclusion, there were no significant changes in esodeviation after unilateral IO-weakening surgery, but bilateral IO-weakening surgery can result in a transient increase in esodeviation in patients with refractive accommodative esotropia.

\section{Conflict of Interest}

No potential conflict of interest relevant to this article was reported.

\section{References}

1. Kushner BJ. Multiple mechanisms of extraocular muscle “overaction". Arch Ophthalmol 2006;124:680-8.

2. Wright KW, Strube YN J. Strabismus surgery. In: Wright KW, Hengst TC, Spiegel PH. Pediatric ophthalmology and strabismus. 3rd ed. [place unknown]: Springer Science \& Business Media; 2013. p. 379-80. 
3. Rajavi Z, Molazadeh A, Ramezani A, Yaseri M. A randomized clinical trial comparing myectomy and recession in the management of inferior oblique muscle overaction. $J$ Pediatr Ophthalmol Strabismus 2011;48:375-80.

4. Ghazawy S, Reddy AR, Kipioti A, et al. Myectomy versus anterior transposition for inferior oblique overaction. $J A A$ POS 2007;11:601-5.

5. Parks MM. Inferior oblique weakening procedures. Int Ophthalmol Clin 1985;25:107-17.

6. Asim AA, Hashmani S, Jamil MA, Zaheer CM. Graded recession for primary inferior oblique overaction. Pak $J$ Ophthalmol 2012;28:122-6.

7. Urist MJ. Surgical treatment of esotropia with bilateral elevation in adduction. AMA Arch Ophthalmol 1952;47:220-47.

8. Shin JH, Paik HJ. Effect of inferior oblique weakening procedures combined with surgery for intermittent exotropia. J Korean Ophthalmol Soc 2015;56:249-53.

9. Stager DR, Parks MM. Inferior oblique weakening procedures. effect on primary position horizontal alignment. Arch Ophthalmol 1973;90:15-6.

10. Taylan Sekeroglu H, Dikmetas O, Sanac AS, et al. Inferior oblique muscle weakening: is it possible to quantify its effects on horizontal deviations? J Ophthalmol 2012;2012.

11. Chang BL, Yang SW. Inferior oblique overaction. J Korean Ophthalmol Soc 1988;2:77-81.

12. Tommila V, Valle O. Effect of inferior oblique muscle recession on horizontal deviation. Acta Ophthalmol (Copenh) 1968;46:779-84.

13. Rosenbaum AL, Santiago AP. Chief complaint, history, and physical examination. In: Rosenbaum AL, Santiago AP. Clinical strabismus management: principles and surgical techniques. 1st ed. Philadelphia: Saunders; 1999. p. 17.
14. Wright KW. Inferior oblique muscle weakening procedures. In: Wright KW, Farzavandi S, Thompson LS. Color atlas of strabismus surgery. 3rd ed. New York: Springer; 2007. p. 166.

15. Parks MM, Parker JE. Atlas of strabismus surgery. 1st ed. Philadelphia: Harper \& Row; 1983. p. 167.

16. Fink WH. Oblique muscle surgery from the anatomic viewpoint. Am J Ophthalmol 1951;34:261-81.

17. Kim WJ, Kim MM. Accommodative esotropia who needs spectacles for good ocular alignment after refractive shift below+ 2.00 diopters. Korean J Ophthalmol 2014;28:41722.

18. Mohan K, Sharma A. Long-term treatment results of accommodative esotropia. J AAPOS 2014;18:261-5.

19. Moon HS, Paik HJ. Long-term follow-up of refractive accommodative esotropia: decompensation and cessation of spectacle use. J Korean Ophthalmol Soc 2012;53:553-8.

20. Ludwig IH, Imberman SP, Thompson HW, Parks MM. Long-term study of accommodative esotropia. J AAPOS 2005;9:522-6.

21. Watanabe-Numata K, Hayasaka S, Watanabe K, et al. Changes in deviation following correction of hyperopia in children with fully refractive accommodative esotropia. Ophthalmologica 2000;214:309-11.

22. Wilson ME, Parks MM. Primary inferior oblique overaction in congenital esotropia, accommodative esotropia, and intermittent exotropia. Ophthalmology 1989;96:950-5.

23. Cakir B, Bursali O, Ozmen S, et al. Factors influencing stereopsis in patients with both refractive accommodative esotropia and amblyopia. Int ophthalmol 2019;39:1263-7.

24. Read JC. Stereo vision and strabismus. Eye (Lond) 2015; 29:214-24. 